\title{
FOCO
}

\section{Estratégias de abordagem ao Cuidador Familiar: Promovendo o cuidado de si $^{1}$}

\author{
Stefanie Griebeler Oliveira ${ }^{2}$ \\ Cristiana Rodrigues Silveira Machado ${ }^{3}$ \\ Taciana Py de Oliveira Osielski ${ }^{4}$ \\ Aline Daiane Leal de Oliveira ${ }^{5}$ \\ Julieta Carriconde Fripp ${ }^{6}$ \\ Isabel Cristina de Oliveira Arrieira ${ }^{7}$ \\ Zayanna Christine Lopes Lindôso ${ }^{8}$
}

\begin{abstract}
Objetivo: relatar sobre as estratégias de abordagem ao cuidador familiar proporcionadas pelo projeto de extensão Um olhar sobre o cuidador familiar: Quem cuida merece ser cuidado. Métodos: Os encontros, realizados semanalmente, foram sistematizados com focos diferentes. No primeiro, o enfoque ocorreu na história do cuidador familiar e elaboração de genograma e ecomapa. No segundo, utilizou-se um vídeo com imagens para disparar reflexões sobre o cotidiano do cuidador. No terceiro, foram abordados desafios, potencialidades e fragilidades da experiência do cuidador, e no quarto, as intervenções planejadas foram executadas. Diversos instrumentos foram utilizados para coleta de dados, avaliação da situação de saúde e sobrecarga possibilitando o planejamento de intervenções junto ao cuidador. Resultados: A escuta terapêutica, o genograma e ecomapa, a identificação das fases do processo de cuidar, permitiram identificar que os cuidadores apresentaram sintomas de estresse e sobrecarga, privações pessoais e sociais em detrimento da dedicação ao processo de cuidar no domicílio, ficando sem tempo para cuidar de si. Conclusões: A Enfermagem e a Terapia Ocupacional na prática interdisciplinar desenvolveram ações educativas, possibilitando um espaço para os cuidadores refletirem sobre si e suas práticas, ajudando-os na elaboração de suas preocupações e emoções, uma vez que ao falar, eles perceberam suas formas de enfrentamento.
\end{abstract}

Palavras-chave Cuidado ao cuidador; serviços de assistência domiciliar; técnicas de si.

${ }^{1}$ Projeto de extensão "Um olhar sobre o cuidador familiar: quem cuida merece ser cuidado" da Universidade Federal de Pelotas (UFPEL), financiado pelo PROEXT-2016.

${ }^{2}$ Enfermeira. Doutora em Enfermagem. Docente da Faculdade de Enfermagem da Universidade Federal de Pelotas (UFPEL)

${ }^{3}$ Terapeuta Ocupacional. Bolsista PROEXT-2016 - Universidade Federal de Pelotas (UFPEL)

${ }^{4}$ Acadêmica do Curso de Terapia Ocupacional da UFPEL. Bolsista PROEXT-2016.Universidade Federal de Pelotas (UFPEL)

${ }^{5}$ Acadêmica de Enfermagem. Bolsista PROEXT-2016 - Universidade Federal de Pelotas (UFPEL)

${ }^{6}$ Médica Paliativista. Doutora em Ciências. Docente da Faculdade de Medicina, Coordenadora Geral Programa Melhor em Casa SADUFPEL - Pelotas junto ao Ministério da Saúde, servidora TAE atuando no Hospital Escola da UFPEL.Universidade Federal de Pelotas (UFPEL)

7 Enfermeira. Doutora em Ciências. Docente do Curso de Enfermagem da Universidade Católica de Pelotas (UCPEL) e coordenadora técnica dos serviços de assistência domiciliar do Hospital Escola da UFPEL.Universidade Federal de Pelotas (UFPEL)

8 Terapeuta Ocupacional. Doutora em Gerontologia Biomédica. Docente do Curso de Terapia Ocupacional da UFPEL.Universidade Federal de Pelotas (UFPEL) 


\title{
Approaching Strategies Towards The Family Caregiver: Promoting The Care Of The Self
}

\begin{abstract}
Objective: to report approaching strategies towards the family caregivers provided by the extension projects "Looking for the family caregiver: who cares deserves to be cared for". Methods: the meetings performed weekly were systematized with a different focus. In the first one, the focus was on the family caregiver's history and genogram and ecomap creation. In the second one, a video with images was used in order to trigger reflections about the daily activities of the caregiver. In the third one, challenges, potentialities, and fragilities about the caregiver's experience were approached, and the planned interventions executed. Diversified tools were used for data collection, evaluation of health status and overburden, which enabled planning the interventions within the caregiver. Results: active listening, genogram, and ecomap, the identification of grieving phases allowed identifying that the caregivers present symptoms of distress and overburden, personal and social privations to the detriment of the care performed at home, getting no time to care of their selves. Conclusions: Nursing and Occupational Therapy, during the interdisciplinary practice, developed educative actions, which provided a space for the caregivers to ponder about them and their practices, also helping them elaborating their worried and emotions, for since they talk, they realize their coping strategies.
\end{abstract}

Keywords: Care to the Caregiver; Home care Services; Self-techniques.

\section{Introdução}

A atenção domiciliar como extensão hospitalar surgiu em 1947 no Hospital de Montefiore em Bronx (Estados Unidos), pensada como forma de abreviar a alta hospitalar. A importância da assistência domiciliar nos Estados Unidos cresceu a partir da década de 1980 com o surgimento da Síndrome da Imunodeficiência Adquirida (SIDA). A necessidade de encontrar maneiras econômicas mais efetivas levaram à exploração da atenção domiciliar também em outros países ocidentais (FEUERWERKER; MERHY, 2008). No Brasil, a primeira forma organizada de assistência domiciliar foi o Serviço de Assistência Médica Domiciliar de Urgência (SAMDU), criado em 1949 (FEUERWERKER; MERHY, 2008; OLIVEIRA, 2014). No início da década de 1990, seguindo uma tendência mundial, surgiu o serviço organizado na forma de cuidado domiciliar (Home Care), concentrado em empresas privadas e em grandes centros. Assim, mesmo sem haver uma política formal ampla de financiamento de práticas assistenciais voltadas à desospitalização, inúmeras organizações de saúde vêm instituindo práticas baseadas na oferta de intervenções terapêuticas no interior do domicílio do usuário conhecido como Atenção domiciliar (AD), que compreende o conjunto de atividades prestadas no domicílio a indivíduos clinicamente estáveis que exijam intensidade de cuidados de menor complexidade que no ambiente hospitalar (FEUERWERKER; MERHY, 2008).

Isto ocorreu e tomou importância devido à crescente lotação de pessoas internadas nos hospitais, o alto risco de infecções e o aumento epidemiológico das doenças crônico-degenerativas, que necessitam de cuidados em longo prazo (WORLD HEALTH ORGANIZATION, 2000). O crescimento da AD no Brasil ainda é recente (BRASIL, 1997; 1998; 2006; 2013; 2016), ocorrendo 
a prestação de serviços tanto no setor privado, quanto no setor público. A atenção domiciliar é regulada pelo Sistema Único de Saúde desde 2011, com o Programa Melhor em Casa, o qual propõe a realização de visitas semanais a pacientes crônicos, pós-operatórios, dentre outras comorbidades. É constituído por Equipe Multiprofissional de Atenção Domiciliar (EMAD) e Equipe Multiprofissional de Apoio (EMAP). São mais de 900 equipes implementadas atualmente no Brasil (BRASIL, 2016).

Porém, para que aconteça esta forma de cuidado ao paciente com doenças crônicas ou em situação de terminalidade, é imprescindível a presença de alguém para realizá-lo, fazendo com que apareça o papel de cuidador. Sem dúvida, os benefícios da atenção domiciliar são reconhecidos pelos pacientes e famílias, devido ao enfermo retornar à sua casa protegido de infecções hospitalares (CORDEIRO, 2017; OLIVEIRA et al, 2015a; OLIVEIRA P. et al 2016; OLIVEIRA; KRUSE, 2017; SILVA et al, 2014). No entanto, é preciso atentar para as questões envolvidas a esta família que executa os cuidados, assumindo o papel institucional de promover a recuperação, o conforto, o alívio do sofrimento, com apoio e controle da equipe multiprofissional de atenção domiciliar (OLIVEIRA; KRUSE, 2017), pois tais cuidadores, tornam-se suscetíveis, por exemplo, a sobrecarga emocional e a privações de necessidades básicas como sono e boa alimentação, vivendo em isolamento social por ficar em torno do paciente e longe de suas atividades ocupacionais (ALPTEKIN et al., 2010; CAMERON, et al., 2002; KUO; OPERARIO; CLUVER, 2012; OLIVEIRA et al, 2015a; OLIVEIRA, S.G et al, 2016; TSHILILO; DAVHANA, 2009). As causas deste sofrimento podem ser emocionais, físicas, sociais e financeiras (VELLEDA; SARTOR; OLIVEIRA, 2014).

Como o programa de $\mathrm{AD}$ visa prestar cuidados à saúde do paciente no domicílio e compreendendo a responsabilidade do cuidador neste contexto, houve a necessidade de ampliar as estratégias de abordagem a ele, que é fundamental na recuperação e no acompanhamento do paciente. $\mathrm{O}$ objetivo deste trabalho é relatar sobre as estratégias de abordagem ao cuidador familiar proporcionadas pelo projeto de extensão Um olhar sobre o cuidador familiar: Quem cuida merece ser cuidado.

\section{Metodologia}

Trata-se de um relato de experiência com reflexões das estratégias de abordagem ao cuidador familiar, propiciadas a partir do método do projeto de extensão "Um olhar sobre o cuidador familiar: Quem cuida merece ser cuidado", que teve sua elaboração em meados de 2014, e Revista Extensão em Foco, nº 13, Jan/ Jul (2017) p. 135 - 148. 
início de execução em junho de 2015. É composto e executado por acadêmicos da Faculdade de Enfermagem e do Curso de Terapia Ocupacional da Universidade Federal de Pelotas (UFPEL) em parceria com o Serviço de Atenção Domiciliar do Hospital Escola-UFPEL/Empresa Brasileira de Serviços Hospitalares (SAD-HE-UFPEL/EBSERH), e oportunizou um espaço de escuta terapêutica e cuidado de si, por meio de visitas domiciliares realizadas aos cuidadores familiares. Tal projeto, foi financiado pelo Programa de Apoio à Extensão Universitária (PROEXT)-2016.

Foram convidados, para participar do projeto, cuidadores familiares de pacientes crônicos ou em situação de terminalidade vinculados ao SAD-HE-UFPEL/EBSERH, composto pelos Programas de Internação Domiciliar Interdisciplinar (PIDI) e Melhor em Casa da cidade Pelotas/RS, maiores de 18 anos.

O PIDI, criado em 2005, oferece cuidado domiciliares para pacientes com diagnóstico de câncer e que são encaminhados pela rede de saúde de Pelotas. Possui atualmente duas equipes multiprofissionais, sendo cada uma responsável pelo cuidado de dez pacientes concomitantes. As visitas são realizadas duas vezes ao dia (ARRIEIRA et al, 2009), a maioria dos pacientes apresenta câncer em estágio avançado, necessitando de cuidados paliativos (FRIPP et al, 2012). Já o programa Melhor em Casa, implantado em 2012, conta com seis equipes que realizam os atendimentos pelo menos uma vez por semana e possuem no máximo 30 pacientes cada, podendo atender até 180 pacientes. Neste serviço, são inclusos usuários dos mais variados agravos à saúde, tais como adultos e crianças, especialmente pacientes com condições crônicas oriundos de hospitais e de unidades básicas de saúde da rede municipal.

O cuidador familiar, foi acompanhado de forma sistematizada por meio da escuta terapêutica ofertada nas visitas domiciliares semanais, totalizando quatro encontros. No primeiro encontro foram coletadas informações sociodemográficas, elaborados genograma e ecomapa e também realizada escuta terapêutica com foco na história da experiência de cuidar.

O genograma consistiu em um diagrama do grupo familiar que representa a sua estrutura interna, por meio de gráficos convencionais genéticos e genealógicos. Teve a finalidade de obter uma visão geral da família, por meio de dados relevantes sobre os relacionamentos ao longo do tempo, e também incluir dados sobre saúde, ocupação, religião, etnia e migrações, entre outras. Assim como o genograma, o ecomapa objetivava oferecer impacto visual, além de conter dados do atual funcionamento familiar, representando os relacionamentos dos integrantes da família com os sistemas mais amplos. O ecomapa evidenciou as relações importantes da família e o mundo (WRIGHT; LEAHEY, 2008), mostrando a complexidade das relações familiares de forma sucinta, para o planejamento de estratégias. Destaca-se ainda que, enquanto o genograma, identifica as Revista Extensão em Foco, nº 13, Jan/ Jul (2017) p. 135 - 148. 
relações e ligações dentro do contexto familiar, o ecomapa é capaz de identificar as relações desta família com o ambiente ao redor, ver como interagem com os demais familiares, vizinhos e que atividades praticam como forma de laser (AGOSTINHO, 2007).

No segundo encontro, um vídeo foi utilizado como disparador reflexivo para a escuta terapêutica, o qual foi constituído por imagens do cotidiano do cuidado (Figura 1), que instigam o cuidador a pensar sobre si próprio e suas práticas diárias. Com essas reflexões, tornou-se possível identificar em que fase de adaptação se encontrava o cuidador.

Figura 1. Imagens elaboradas para o projeto de extensão

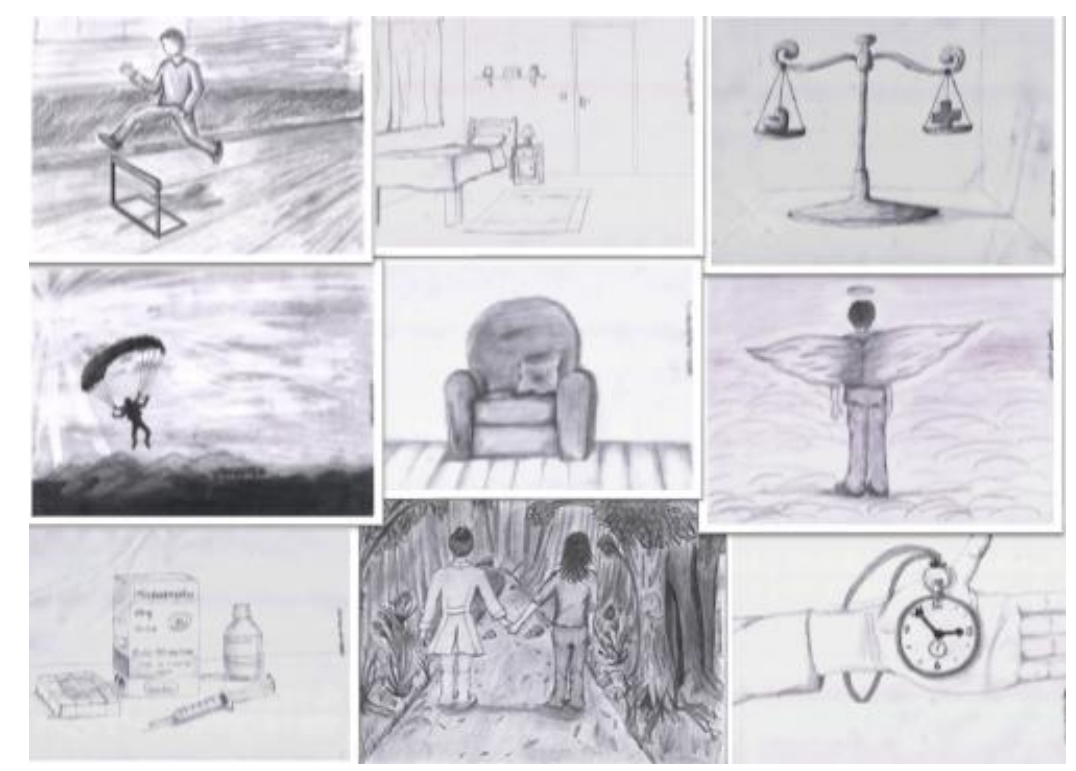

Fonte: (OLIVEIRA et al, 2015b).

As fases de adaptação foram: Negação; Busca de informação; Reorganização e Resolução. A primeira, consistiu em uma reação psicológica de autoproteção, a qual permitiu ao cuidador controlar seus medos e ansiedades, pois faz com que ele se afaste da ameaça e incerteza da doença que afeta seu familiar. Significou um tempo necessário para que o cuidador percebesse as dificuldades que o paciente apresentava. Quando prolongada, tal fase poderia se converter em fuga, impedindo o cuidador de avançar no processo de cuidado. Na segunda, ocorria aceitação da realidade do doente, mas, poderia fazer emergir sentimentos de angústia, raiva, culpa, frustração e vitimização devido a situação que creem não merecer. Já na terceira fase, a chamada reorganização, a vida dos cuidadores se reorganizava com o passar do tempo, mesmo que persistissem alguns sentimentos como raiva, frustração, solidão frente a condição do paciente. Ele sentia que poderia ter mais controle da situação e aceitava as modificações que a situação comportava, pois adequava sua

Revista Extensão em Foco, nº 13, Jan/ Jul (2017) p. 135 - 148. 
vida conforme as necessidades do paciente. Por último, na resolução, os cuidadores apresentavamse mais tranquilos, apesar das dificuldades permanecerem e eram capazes de atender com êxito as demandas dos cuidados presentes e futuros (FERRÉ-GRAU et al., 2011).

No terceiro encontro, foram executadas as intervenções planejadas (FERRÉ-GRAU et al., 2011; OLIVEIRA et al, 2015b) a partir das necessidades detectadas pela classificação da fase de adaptação do processo de cuidar e também pelas narrativas produzidas ao longo dos encontros. O quadro de intervenções foi apresentado nos resultados, uma vez que inicialmente, algumas intervenções foram retiradas de Ferré-Grau et al (2011), e outras, foram elaboradas pelo grupo do projeto de extensão, nas reuniões em que os casos acompanhados eram discutidos. Portanto, compreendeu-se o quadro de intervenções também como um resultado das ações do projeto. Também neste encontro, a escuta terapêutica foi focada nos enfrentamentos vividos, nas dificuldades e fragilidades do processo de cuidar.

Para elaboração das intervenções, as seguintes etapas foram respeitadas: 1) Avaliação do contexto no qual o paciente e cuidador estavam inseridos, considerando as condições socioeconômicas, o espaço físico e o contexto familiar, com o objetivo de identificar se o cuidador poderia se ausentar caso fosse necessário e por quanto tempo; 2) Elaboração de um plano de intervenção com base nas necessidades dos cuidadores.

E por fim, no quarto encontro, a realização e avaliação das intervenções e o feedback das ações desenvolvidas pelo projeto. Sempre que fosse necessário, as visitas ao cuidador eram estendidas em mais encontros.

\section{Resultados e discussão}

\section{Caracterização dos participantes}

Até o momento foram atendidos no projeto 52 cuidadores familiares, sendo 50 mulheres e apenas dois homens. Destes, apenas 14 recebiam ajuda no cuidado com os pacientes, com revezamento nos finais de semana. A idade dos cuidadores variou entre 30 a 76 anos. Apenas nove cuidadores haviam tido anteriormente experiência com cuidado e 20 deles privaram-se de seus trabalhos para assumir esse papel. A maioria dos cuidadores relatou ter o ensino fundamental incompleto, 10 referiu ter ensino superior completo e apenas 1 cuidadora dizer que não era alfabetizada. Todos relataram que não recebiam remuneração pela prestação do cuidado e também que viviam com a renda familiar do paciente beneficiários ou aposentados do Instituto Nacional de Seguridade Social (INSS). Em relação as fases de adaptação da doença, a maioria se encontrava em Revista Extensão em Foco, nº 13, Jan/ Jul (2017) p. 135 - 148. 
fase de Reorganização. E 48 cuidadores queixaram-se sobre a falta de tempo de cuidar de si e de viverem momentos de lazer.

Todos os cuidadores apresentavam ou já apresentaram sintomas de estresse e depressão como irritabilidade, sensação de vazio, impaciência e intolerância, tristeza seguido de choro e alguns pensamentos suicidas.

Sobre a importância dos atendimentos voltados para o cuidador, 50 deles responderam que foi de grande importância, e recomendaram a ampliação de outros programas em prol desta população. Apenas duas cuidadoras desistiram dos atendimentos, por motivos diversos, sendo um deles a falta de tempo para se organizar e receber os acadêmicos.

\section{Estratégias de abordagem ao cuidador: potencialidades}

A escuta terapêutica, o genograma e ecomapa, a identificação das fases do processo de cuidar, permitiram identificar que os cuidadores apresentavam sintomas de estresse e sobrecarga, privações pessoais e sociais em detrimento da dedicação ao processo de cuidar no domicílio, ficando sem tempo para cuidar de si.

A escuta terapêutica atravessou todos os encontros, abordando temas como a história e experiências de cuidar, enfrentamentos, desafios, práticas de alívio, entre outros. A partir disso, o cuidador foi refletindo sobre sua fala e acontecimentos, aliviando sua sobrecarga e pensando como ele se modificou ao longo desse processo de cuidado. Além disso, se sentiu valorizado, por ter esse espaço de fala e escuta em que o mesmo não experimentou julgamento, por parte dos acadêmicos, em relação aos seus pronunciamentos.

Neste sentido, a escuta terapêutica consistiu na habilidade do profissional em usar seu conhecimento para ajudar a pessoa com tensão temporária a conviver com outras pessoas e ajustarse nas situações que não poderiam ser modificadas, bem como, a superar seus bloqueios à autorealização para enfrentar seus problemas (PONTES; LEITÃO; RAMOS, 2008). A comunicação tornou-se um grande fator de humanização na atenção à saúde por favorecer o entendimento e a reciprocidade dos conteúdos que envolveram o significado da doença e as atitudes coerentes perante o tratamento e a promoção da saúde e da vida (BERTACHINI, 2012).

O genograma e o ecomapa (Figura 2), elaborados junto com o cuidador familiar no primeiro encontro, foram instrumentos que facilitaram a avaliação da estrutura familiar. Com ele, foi possível identificar os laços fortes e as formas de apoio, entre eles: familiares, religião, apoio social da comunidade, rede de apoio a saúde e lazer. O apoio mais frequente foi o da família, onde Revista Extensão em Foco, nº 13, Jan/ Jul (2017) p. 135 - 148. 
identificou-se cônjuges, filhos, irmãos, sobrinhos, ex companheiros e o próprio paciente. Na religião, percebeu-se que a citação desta, poderia estar relacionada com a finitude da vida e os sentimentos que essa desperta no paciente e em sua família. Já o apoio social na comunidade estava relacionado aos vizinhos, aos amigos e a própria comunidade. A rede de apoio a saúde que foi mencionada era composta pelo PIDI, Unidade Básica de Saúde, Melhor em casa, hospital, Associação de Apoio a Pessoas com Câncer e profissionais de saúde. Por fim, o lazer, foi caracterizado por assistir televisão, ler, acessar as redes sociais e assistir filmes

Figura 2. Exemplo de genograma e ecomapa elaborado de um dos cuidadores

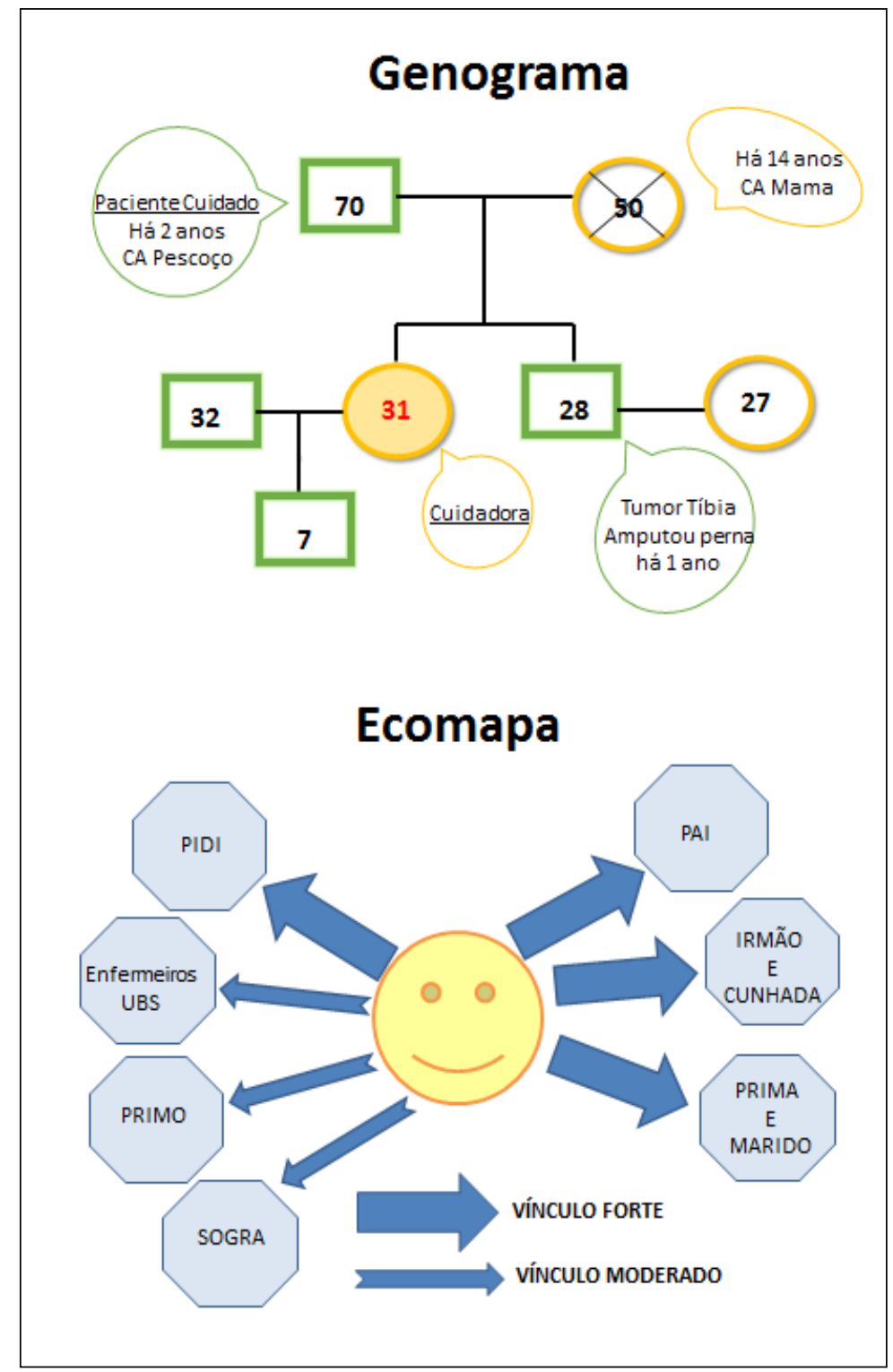

Fonte: (OLIVEIRA et al, 2015b).

Revista Extensão em Foco, nº 13, Jan/ Jul (2017) p. 135 - 148. 
O vídeo composto por imagens do cotidiano (Figura1), que foi utilizado no segundo encontro como disparador reflexivo para a escuta terapêutica, propiciou que os mesmos relacionassem com experiências de cuidar. Para alguns, poderiam ser imagens simples, mas para estes cuidadores elas tiveram significado, o que permitiu que falassem de suas angústias e anseios. Por exemplo, o anjo representou o apoio da família, dos profissionais de saúde, e também, a própria ação de cuidar. A balança, foi significada como o equilíbrio que o cuidador precisava buscar entre razão e emoção, para prosseguir nas ações do cuidado. O relógio teve diversas representações. Uma delas era relacionada ao horário dos medicamentos e de outros cuidados. Outra, muito frequente, foi em relação ao tempo que se ocupa para o cuidado do outro, e o pouco tempo para si.

Utilizar recursos audiovisuais como instrumento para auxiliar durante a comunicação permite aprofundar em questões vivenciadas pelas pessoas abordadas (VALE, 2014), despertando reflexões e questionamentos (LISBOA; PIRES, 2010). No caso dos cuidadores, essa ferramenta instigou pensamentos sobre as atribuições e emoções relacionadas ao cuidado com o familiar doente, rotina que muitas vezes foi reproduzida sem espaço para descanso, gerando sentimentos negativos (ANJOS; ZAGO, 2014) que devem ser considerados e discutidos.

Ainda no segundo encontro, foi possível identificar em qual fase de adaptação no processo de cuidado ele se encontra para o planejamento das intervenções que foram realizadas (Figura 3). Algumas destas intervenções, foram retiradas do Guía de cuidados de enfermeira: cuidar al cuidador em atención primária (intervenções:1,5-6,9,13-24). As demais foram elaboradas pelo próprio grupo de execução do projeto, enquanto se discutia os casos dos cuidadores que estavam sendo acompanhados, conforme suas necessidades.

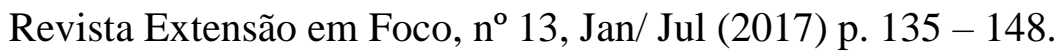


Quadro 1. Quadro de intervenções sugeridas e realizadas (*intervenções do campo da enfermagem; ** intervenções do campo da Terapia Ocupacional).

\begin{tabular}{|c|c|}
\hline \multicolumn{2}{|c|}{ INTERVENÇÕES SUGERIDAS E REALIZADAS } \\
\hline 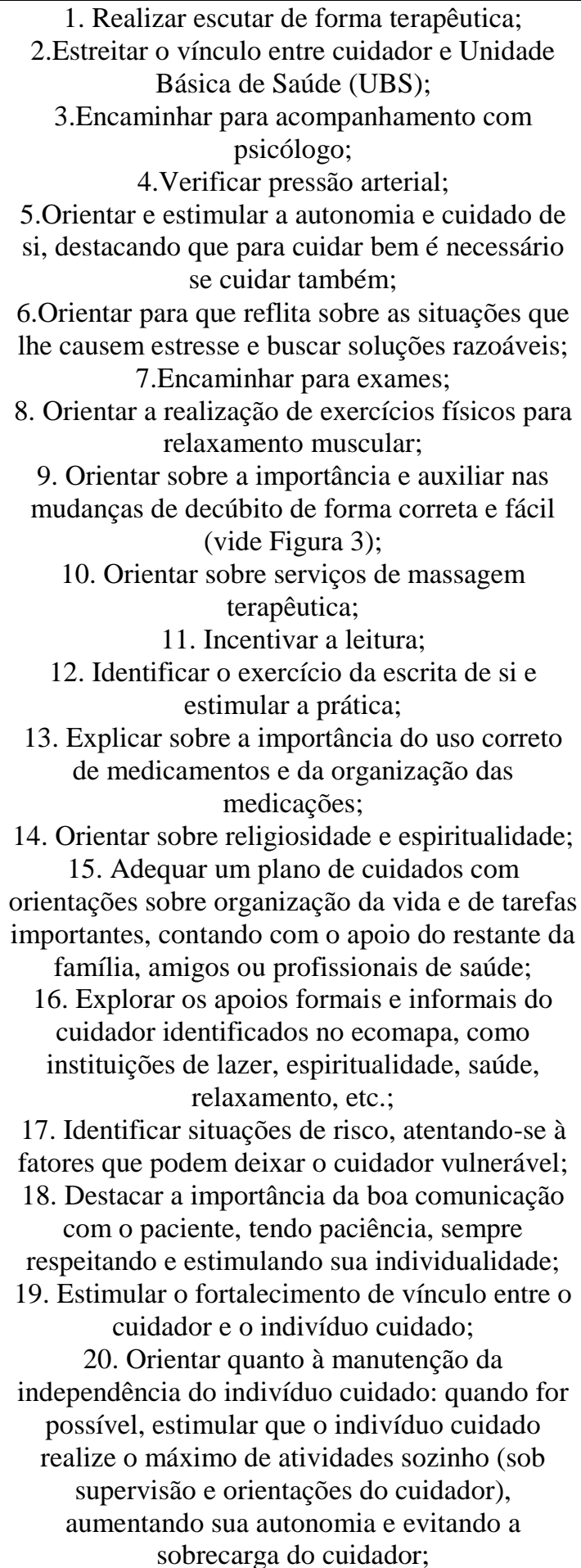 & 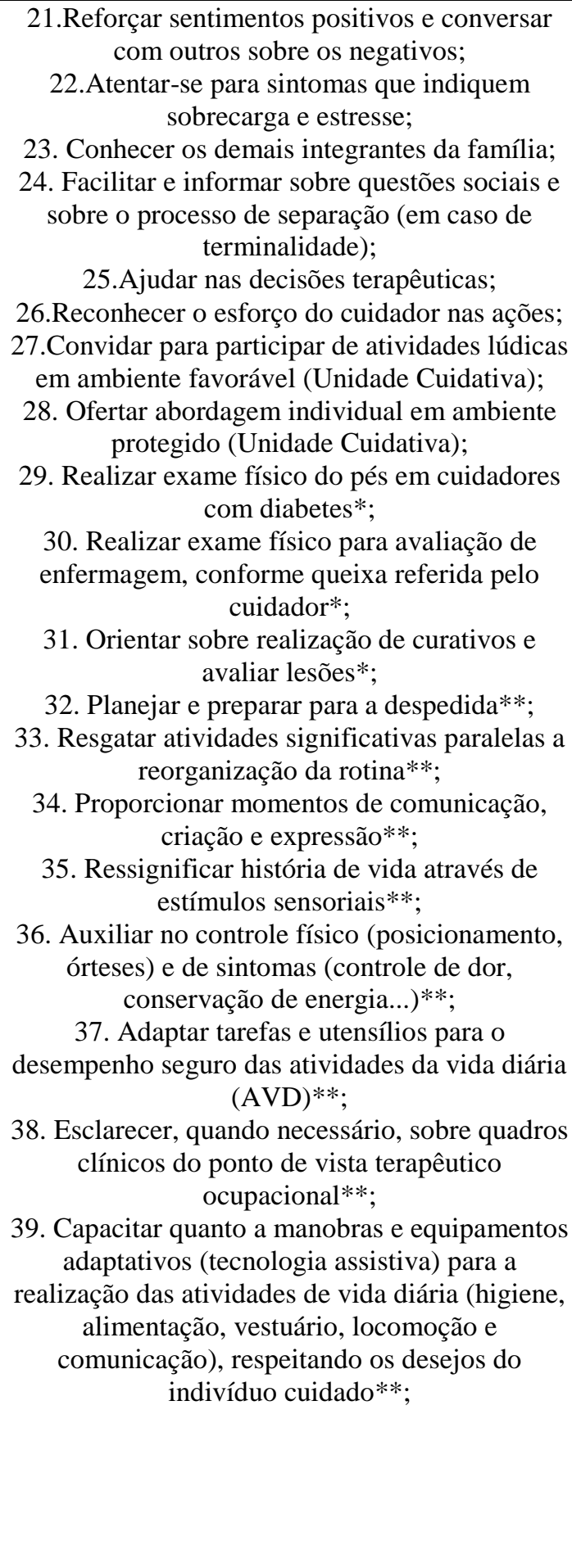 \\
\hline
\end{tabular}

Fonte: Dados projeto de extensão (OLIVEIRA et al, 2015b). 
À medida que as intervenções eram realizadas, desde a identificação das necessidades, estas eram avaliadas tanto pelo cuidador, receptor destes cuidados, quanto pelos acadêmicos que buscavam identificar a melhoria na organização do processo de cuidar nos momentos das visitas. Assim como muitas intervenções foram elaboradas nas reuniões do grupo de execução do projeto, a avaliação dos resultados de tais intervenções também foram discutidas neste espaço coletivo.

Segundo Santos et al (2011) a sobrecarga do cuidador é definida como o conjunto de problemas físicos, psicológicos, emocionais, sociais e financeiros. Em geral, o cuidador é o único responsável pela rede de cuidados necessários ao sujeito, e, é comum o desconhecimento sobre como lidar adequadamente com eles e consigo mesmo, surge então à necessidade de orientação.

Foi observado que a maioria dos cuidadores familiares não teve escolha em relação a assumir esse papel, uma vez que, eram geralmente de família com renda baixa, impedindo a contratação de um cuidador profissional. Nesse sentido, acabaram assumindo tal responsabilidade, sem remuneração e muitas vezes sem nenhum reconhecimento. Abriram mão do seu lazer, dos seus amigos, dos seus empregos e até do tempo para o cuidado de si.

Salienta-se então uma reflexão para maiores projetos e pesquisas neste ramo da saúde, possibilitando maior experiência a acadêmicos e maior conforto a essas pessoas que se encontram em um momento muito delicado de suas vidas.

\section{Considerações finais}

O projeto de extensão vai ao encontro das políticas de saúde, em especial as voltadas para a atenção domiciliar, que vêem se consolidando no cenário de saúde atual. Convergem com a necessidade de acolher este novo sujeito, o cuidador familiar, fundamental para a modalidade de cuidado e para o acompanhamento do paciente no domicílio. Deste modo, estratégias de abordagem ao cuidador são necessárias e fundamentais para o alívio da sobrecarga que este enfrenta.

Cabe então reforçar a importância do projeto de extensão relatado, pois, partindo das práticas interdisciplinares da Enfermagem e da Terapia Ocupacional, acredita-se que os cuidadores familiares foram beneficiados com o espaço de escuta, onde puderam expressar suas preocupações e emoções, bem como, pelas intervenções que foram realizadas. A oferta de espaço de escuta terapêutica e a utilização das diversas estratégias de abordagem ao cuidadores familiares, permitiram aos mesmos realizarem reflexões e falarem de si, para que possam elaborar suas próprias técnicas de cuidado e também compartilhar com acadêmicos, docentes e profissionais de saúde como são seus meios de enfrentamento e adaptações. Para os acadêmicos, oportuniza o Revista Extensão em Foco, nº 13, Jan/ Jul (2017) p. 135 - 148. 
desenvolvimento de um olhar sensibilizado, humanizado, voltado para o cuidado integral, com inserção do cuidador no processo de cuidar.

\section{Referências}

AGOStinhO, M. Ecomapa. Revista Portuguesa de Clinica Geral, Lisboa, n.23, p. 327-330, 2007. Disponível em: http://eventos.fecam.org.br/arquivosbd/paginas/1/0.307825001366390062_ecomapa.pdf. Acesso em: 29 jun. 2015.

ALPTEKIN, S. et al. Characteristics and quality of life analysis of caregivers of cancer patients. Medical Oncology, Totowa, v. 27, n.3, p. 607-617, sep. 2010.

ANJOS, A. C. Y.; ZAGO, M. M. F. Ressignificação da vida do cuidador do paciente idoso com câncer. Revista Brasileira de Enfermagem, Brasília, v.67, n.5, p.752-8, 2014.

ARRIEIRA, I. C. O. et al. Programa de Internação Domiciliar Interdisciplinar oncológico: Metodologia de Trabalho. Ciência, Cuidado e Saúde, Maringá, v. 8 (suplem), p. 104-109, dez. 2009. Disponível em: http://www.periodicos.uem.br/ojs/index.php/CiencCuidSaude/article/view/9725/553 Acesso em: 26 mar 2016.

BERTACHINI, L. A Comunicação Terapêutica como fator de humanização na Atenção Primária. O Mundo de Saúde, São Paulo, n. 36, v. 3, p. 507-520, 2012.

BRASIL. Ministério da Saúde. Portaria $n^{\circ} 1.892$, de 18 de dezembro de 1997. Incorpora a modalidade Internação Domiciliar ao Sistema único de Saúde. Diário Oficial [da] República Federativa do Brasil, Brasília, DF, 22 dez. 1997. Seção I, p.38.

BRASIL. Ministério da Saúde. Portaria n ${ }^{\circ}$ 2.416, de 23 de março de 1998. Estabelece requisitos para credenciamento de Hospitais e critérios para realização de internação domiciliar no SUS. Diário Oficial [da] República Federativa do Brasil, Brasília, DF, 26 mar.1998. Seção I, p.106.

BRASIL. Ministério da Saúde. Portaria $\mathrm{n}^{\circ}$ 2.529, de 19 de outubro de 2006. Institui a Internação Domiciliar no âmbito do SUS. Diário Oficial [da] República Federativa do Brasil, Brasília, DF, 20 out. 2006. Seção I, p.145-148.

BRASIL. Ministério da Saúde. Portaria $\mathrm{n}^{\circ}$ 1.208, de 18 de junho de 2013. Dispõe sobre a integração do Programa Melhor em Casa (Atenção Domiciliar no âmbito do SUS) com o Programa SOS Emergências, ambos inseridos na Rede de Atenção às Urgências. Diário Oficial [da] República Federativa do Brasil, Brasília, DF, 19 jun. 2013. Seção I, p.37.

BRASIL. Ministério da Saúde. Portaria $\mathrm{n}^{\circ}$ 825, de 25 de abril de 2016. Redefine a atenção domiciliar no âmbito do Sistema Único de Saúde (SUS) e atualiza as equipes habilitadas. Diário Oficial [da] União, Brasília, DF, 25 abr. 2016. 
CAMERON, J. I. et al. Lifestyle interference and emotional distress in family caregivers of advanced cancer patients. Cancer, Philadelphia, v.94, n.2, p.521-527, jan. 2002.

CORDEIRO, F.R. O retorno ao domicílio em cuidados paliativos: interface dos cenários brasileiro e francês. Tese (Doutorado em Enfermagem) - Escola de Enfermagem, Universidade Federal do Rio Grande do Sul, Porto Alegre. 2017.

FERRÉ-GRAU et al. Guía de cuidados de enfermeira: cuidar al cuidador em atención primária. Sevilla: Publidisa, 2011.

FEUERWERKER, L.C.M.; MERHY, E.E. A contribuição da atenção domiciliar para a configuração de redes substitutivas de saúde: desinstitucionalização e transformação de práticas. Revista Panamerica Salud Publica, Washington, v.24, n.3, p.180-188, 2008.

FRIPP, J.C.; FACCHINI, L.A.; SILVA, S.M. Caracterização de um programa de internação domiciliar e cuidados paliativos no Município de Pelotas, Estado do Rio Grande do Sul, Brasil: uma contribuição à atenção integral aos usuários com câncer no Sistema Único de Saúde, SUS. Epidemiologia e Serviços de Saúde, Brasília, v.21, n.1, p.69-78, jan./mar. 2012.

KUO, C.; OPERARIO, D.; CLUVER, L. Depression among carers of AIDS-orphaned and otherorphaned children in Umlazi Township, South Africa. Global Public Health, London, v.7, n.3, p.253-260, mar. 2012.

LISBOA, M.; PIRES, G. L. Reflexões sobre a imagem e a fotografia: possibilidades na pesquisa e no ensino da educação física. Motrivivência, Florianópolis, v.22, n.34, p.72-86, 2010.

OLIVEIRA, P.M. et al. Visão do familiar cuidador sobre o processo de morte e morrer no domicílio. Revista Baiana Enfermagem, Salvador, v.30, n.4, p.1-11, out./dez. 2016.

OLIVEIRA, S.G. Melhor em casa? Um estudo sobre a atenção domiciliar. Tese (Doutorado em Enfermagem)- Escola de Enfermagem, Universidade Federal do Rio Grande do Sul, Porto Alegre. 2014.

OLIVEIRA, S.G.; KRUSE, M.H.L.; SARTOR, S.F.; ECHEVARRÍA-GUANILO, M.E. Enunciados sobre la atención domiciliaria en el panorama mundial: revisión narrativa. E global, Múrcia, v.14, n.3, p.375-389, jul. 2015a.

OLIVEIRA, S.G et al. Um olhar sobre o cuidador familiar: quem cuida merece ser cuidado. Pelotas: Universidade Federal de Pelotas; Pró-Reitoria de Extensão e Cultura; Faculdade de Enfermagem, 2015b.

OLIVEIRA, S.G. et al. Representações sociais do cuidado de doentes terminais no domicílio: o olhar do cuidador familiar. Aquichán, Bogotá, v.16, n.3, p.359-369, 2016.

OLIVEIRA, S.G.; KRUSE, M.H.L. Melhor em Casa: dispositivo de segurança. Texto \& Contexto Enfermagem, Florianópolis, v.26, n.1, p.1-9, 2017. 
PONTES, A.C; LEITÃO, I.M.T; RAMOS, I.C. Comunicação Terapêutica em Enfermagem: instrumento essencial do cuidado. Revista Brasileira de Enfermagem, Brasília, n. 3, v. 61, 312-318. 2008.

SANTOS, L. et al. Intervenções de Grupo para Sobrecarga de Cuidadores de Pacientes com Demência: Uma Revisão Sistemática. Revista Psiquiátrica Clínica, Coimbra, v.38, n.4, p.161-167, 2011.

SILVA, K.L. et al. Desafios da atenção domiciliar sob a perspectiva da redução de custos/racionalização de gastos. Revista de Enfermagem da UFPE on line, v.8, n.6, p.1561-1567, jun. $2014 . \quad$ Disponível em: http://www.revista.ufpe.br/revistaenfermagem/index.php/revista/article/view/4894 Acesso em: 24 jan. 2017.

TSHILILO, A. R.; DAVHANA-MASELESELE, M. Family experiences of home caring for patients with HIV/AIDs in rural Limpopo Province, South Africa. Nursing \& Health Sciences, Melbourn, v. 11, n. 2, p. 135-143, jun. 2009.

VALE, A. F. C. Etnografia, sexualidade e imagem: reflexões sobre o uso do vídeo na pesquisa antropológica. In: Encontro Anual da ANPOCS, 2014. Anais do $38^{\circ}$ Encontro Anual da ANPOCS. São Paulo: Associação Nacional de Pós-Graduação e Pesquisa em Ciências Sociais, 2014. p.1-23.

VELLEDA, K. L.; SARTOR, S. F.; OLIVEIRA, S. G. Cuidados paliativos: uma reflexão sobre alternativas em prol do cuidador familiar. In: Seminário Internacional de Bioética e Saúde Pública, 2, 2014, Santa Maria. Anais: II Seminário Internacional de Bioética e Saúde Pública e II Simpósio Internacional de Ética na Pesquisa, 4, 5, 6 e 7 de junho de 2014, Santa Maria. p.227-234.

WORLD HEALTH ORGANIZATION. Home-based long-term care: report of a WHO study group. Geneva, 2000.

WRIGHT, L. M.; LEAHEY, M. Enfermeiras e famílias: um guia para avaliação e intervenção na família. São Paulo: Roca, 2008.

Revista Extensão em Foco, nº 13, Jan/ Jul (2017) p. 135 - 148. 